\title{
Does a gluten-free diet determine the efficacy of sotagliflozin in patients with concomitant type 1 diabetes mellitus and celiac disease?
}

\author{
Dimitrios loannis Patoulias ${ }^{1,2}$, Petros Keryttopoulos ${ }^{2}$ \\ ${ }^{1} 2^{\text {nd }}$ Propaedeutic Department of Internal Medicine, Aristotle University of Thessaloniki, General Hospital Hippokration, \\ Thessaloniki, Greece \\ 2Department of Internal Medicine, General Hospital of Veria, Veria, Greece
}

Gastroenterology Rev 2018; 13 (3): 249-250

DOI: https://doi.org/10.5114/pg.2018.78291

Address for correspondence: Dimitrios loannis Patoulias MD, Department of Internal Medicine, General Hospital of Veria, 3B M. Alexandrou St, 57010 Veria, Greece, phone: +30 6946900777, e-mail: dipatoulias@gmail.com

Sodium glucose-cotransporter type 2 (SGLT-2) inhibitors constitute a novel class of antidiabetics, approved for the treatment of type 2 diabetes mellitus. There are sufficient data regarding their contribution to significant improvement in the major cardiometabolic parameters $\left(\mathrm{HbA}_{1 \mathrm{c}}\right.$, fasting plasma glucose, blood pressure, lipid profile, body weight), with promising results in cardiovascular and diabetic kidney disease [1-4].

Their insulin-independent mechanism of action makes them an attractive and promising treatment option in patients with type 1 diabetes mellitus, as an adjunct therapy to insulin. Sodium glucose-cotransporter type 2 inhibitors provide significant improvement in two main adverse effects of insulin, namely hypoglycaemic events and weight gain; thus, the discussion on their use in those patients is ongoing.

Sotagliflozin, a dual SGLT1/SGLT2 inhibitor, acts as a glucagon-like peptide 1 secretagogue, as well. The latter makes sotagliflozin an interesting therapeutic option in diabetic patients [5].

Garg et al. reported significant clinical benefits of sotagliflozin when added to insulin in patients with type 1 diabetes, namely improved glycaemic control, along with weight reduction and blood pressure lowering effects, results similar to those provided by Sands et al., despite the substantial differences in the number of involved patients and the short-term evaluation of the efficacy of sotagliflozin $[6,7]$.

We would like to draw attention to a specific point. It is well established that type 1 diabetes and celiac disease share common alleles, leading to frequent concom- itance of the two diseases, both in youths and adults $[8,9]$. Based on the fact that SGLT1 expression has been shown to be absent in patients with untreated celiac disease, normalising after initiation of a gluten-free diet for at least 12 months, it seems reasonable that sotagliflozin, a dual SGLT1/SGLT2 inhibitor, may provide significant therapeutic results in patients with concomitant type 1 diabetes and celiac disease, along with appropriate dietary modification [10]. In other words, sotagliflozin is expected to act only through SGLT2 inhibition in those patients with untreated celiac disease, leading to insufficient glycaemic control. Thus, it is deduced that in patients following a gluten free diet, sotagliflozin will inhibit both SGLT1 and SGLT2 inhibitors, maximizing its glucose lowering properties. Another question that arises is whether sotagliflozin is equally efficient in type 1 diabetic patients with treated celiac disease and in type 1 diabetic patients without celiac disease.

After conjugation of pathophysiologic mechanisms and clinical data, it seems that sotagliflozin is a very promising treatment option. Of course, this is a hypothesis based upon very limited relevant literature, which must be further elucidated. Thus, further clinical trials concerning the use of sotagliflozin in patients with concomitant type 1 diabetes and celiac disease, both treated and untreated, and the achieved glycaemic control are required, in order to reinforce, confirm, or reject this hypothesis.

\section{Conflict of interest}

The authors declare no conflict of interest. 


\section{References}

1. Zinman B, Wanner C, Lachin JM, et al.; EMPA-REG OUTCOME Investigators. Empagliflozin, cardiovascular outcomes, and mortality in type 2 diabetes. N Engl J Med 2015; 373: 2117-28.

2. Wanner C, Inzucchi SE, Lachin JM, et al.; EMPA-REG OUTCOME Investigators. Empagliflozin and progression of kidney disease in type 2 diabetes. N Engl J Med 2016; 375: 323-34.

3. Neal B, Perkovic V, Mahaffey KW, et al.; CANVAS Program Collaborative Group. Canagliflozin and cardiovascular and renal events in type 2 diabetes. N Engl J Med 2017; 377: 644-57.

4. Birkeland KI, Jørgensen ME, Carstensen B, et al. Cardiovascular mortality and morbidity in patients with type 2 diabetes following initiation of sodium-glucose co-transporter-2 inhibitors versus other glucose-lowering drugs (CVD-REAL Nordic): a multinational observational analysis. Lancet Diabetes Endocrinol 2017; 5: 709-17.

5. Zambrowicz B, Ogbaa I, Frazier K, et al. Effects of LX4211, a dual sodium-dependent glucose cotransporters 1 and 2 inhibitor, on postprandial glucose, insulin, glucagon-like peptide 1 , and peptide tyrosine tyrosine in a dose-timing study in healthy subjects. Clin Ther 2013; 35: 1162-73.

6. Garg SK, Henry RR, Banks P, et al. Effects of sotagliflozin added to insulin in patients with type 1 diabetes. N Engl J Med 2017; 377: 2337-48.

7. Sands AT, Zambrowicz, BP, Rosenstock J, et al. Sotagliflozin, a dual SGLT1 and SGLT2 inhibitor, as adjunct therapy to insulin in type 1 diabetes. Diabetes Care 2015; 38: 1181-8.

8. Smyth DJ, Plagnol V, Walker NM, et al. Shared and distinct genetic variants in type 1 diabetes and celiac disease. N Engl J Med 2008; 359: 2767-77.

9. Craig ME, Prinz N, Boyle CT et al. Prevalence of celiac disease in 52,721 youth with type 1 diabetes: international comparison across three continents. Diabetes Care 2017; 40: 1034-40.

10. Laforenza U, Miceli E, Gastaldi G, et al. Solute transporters and aquaporins are impaired in celiac disease. Biol Cell 2010; 102: 457-67.

Received: 12.01 .2018

Accepted: 24.04.2018 